\title{
Dieta de bajo índice glicémico en embarazadas para prevenir macrosomía (ROLO study): Un estudio clínico randomizado
}

\author{
Walsh JM, McGowan CA, Mahony R, Foley ME, McAuliffe FM. BMJ 2012;345:e5605. \\ Análisis crítico: Simone Pohlhammer I. ${ }^{1}$, Claudio Vera P-G., MSc ${ }^{2}$, Jorge Carvajal C., PhD. ${ }^{2}$ \\ ${ }^{1}$ Programa Obstetricia-Ginecología, ${ }^{2}$ División de Obstetricia y Ginecología, Unidad de Medicina Materno-Fetal; \\ Facultad de Medicina, Pontificia Universidad Católica de Chile.
}

\section{RESUMEN (1)}

Objetivo: Determinar si una dieta de bajo índice glicémico durante el embarazo es capaz de reducir la incidencia de macrosomía fetal en una población de riesgo. Diseño: Estudio clínico randomizado, realizado en el Hospital Nacional de Maternidad, Dublín, Irlanda. Participantes: 800 mujeres sin diabetes, todas cursando su segundo embarazo, con el antecedente de un parto previo mayor a $4 \mathrm{~kg}$, durante el periodo comprendido entre enero 2007 y enero 2011. Intervención: Las mujeres fueron randomizadas a no recibir intervención dietaria o a comenzar una dieta con un bajo índice glicémico desde el inicio del embarazo. Resultados principales: El resultado primario fue diferencias en el peso al nacer. El resultado secundario fue la diferencia en la ganancia de peso materno durante el embarazo. Resultados: No se observaron diferencias significativas entre los dos grupos en el peso absoluto al nacer, percentil de peso al nacer o índice ponderal. La ganancia de peso gestacional fue significativamente menor en la rama intervencional (12,2 vs 13,7 kg; diferencia promedio: $-1,3$; $95 \% \mathrm{IC}$ : $-2,4$ a $-0,2 ; p=0,01)$. La tasa de mujeres con intolerancia a la glucosa fue menor en el grupo intervenido $21 \%$ (67/320) comparado con $28 \%$ (100/352) en el grupo control, los que tuvieron tuvieron una glicemia de ayuno mayor o igual a $5,1 \mathrm{mmol} / \mathrm{L}$ o test te tolerancia a la glucosa a la hora mayor a 7,8 $\mathrm{mmol} / \mathrm{L}(p=0,02)$. Conclusiones: Una dieta de baja índice glicémico durante el embarazo no reduce la incidencia de recién nacidos grandes para la edad gestacional en un grupo de riesgo de presentar macrosomía fetal. Sin embargo, si tiene un efecto positivo significativo en reducir el aumento de peso gestacional y la intolerancia a la glucosa materna.

\section{ANÁLISIS DE LA INVESTIGACIÓN}

\section{A. Relevancia clínica de la investigación}

La prevalencia mundial de recién nacido $\geq 4000 \mathrm{~g}$ es aproximadamente el $9 \%$, con grandes variaciones entre los países (2). La macrosomia fetal tiene complicaciones maternas conocidas, como trabajo de parto prolongado o detenido, parto vaginal operatorio, cesárea, laceraciones del canal del parto y hemorragia posparto entre otras. La macrosomía tiene también complicaciones fetales y neonatales dentro de las que podemos destacar distocia de hombros, lesiones de plexo braquial, fractura de clavícula y asfixia, y puede traer riesgos a largo plazo como desarrollo posterior de obesidad, intolerancia a la glucosa o síndrome metabólico (3). Considerando estos antecedentes parece importante reducir el número de fetos macrosómicos en pacientes con factores de riesgo, ya que se ha observado que en ellas el riesgo de recurrencia es de $30-50 \%$ (4). Estudios previos muestran beneficios en reducir la incidencia de macrosomía fetal cuando se realiza una dieta de bajo índice glicémi$\mathrm{co}$, sin embargo, estos estudios son de baja calidad metodológica $(5,6)$. Este estudio es el primer rando- 
mizado y de metodología rigurosa.

\section{B. El estudio (1)}

Diseño: Estudio clínico randomizado realizado en el periodo comprendido entre enero 2007 y enero 2011 en el Hospital Nacional de Maternidad, en Dublín, Irlanda. Pacientes: 800 mujeres sin diabetes, todas cursando su segundo embarazo, con el antecedente de un parto previo mayor a $4 \mathrm{~kg}$. Intervención: 372 mujeres recibieron una sesión de educación dietaria de dos horas, en ella se les instruyó sobre cómo elegir una dieta de bajo índice glicémico, normocalórica. La nutricionista se reunió con las pacientes a las 28 y 34 semanas para reforzar los conceptos y responder preguntas. Comparación: 387 mujeres recibieron control prenatal de rutina. Resultado primario: Diferencias del peso al nacer. Resultado secundario: Diferencia en la ganancia de peso durante el embarazo. Resultados: Como se observa en la Tabla I, no se demostraron diferencias en el resultado primario del estudio. El resultado secundario mostró una reducción significativa en la ganancia de peso durante el embarazo en las mujeres intervenidas.

\section{Análisis Crítico}

Validez interna: Estudio con bajo riesgo de sesgo con descripción apropiada de los criterios de inclusión y exclusión, randomizado, con ocultamiento de la secuencia, similitud de pacientes en ambos grupos, con seguimiento y análisis por intención de tratar.

Un punto relevante en el análisis crítico de esta investigación es que el resultado primario es clínicamente irrelevante: el título del estudio da a entender al lector que el objetivo principal es la reducción de la macrosomía fetal, sin embargo el texto muestra que el resultado primario es la reducción de peso al nacer. En efecto, para el cálculo del tamaño muestral se propone demostrar una reducción de peso de nacimiento de $102 \mathrm{~g}$. Semejante resultado primario no es clínicamente relevante; el resultado relevante es la macrosomía fetal o sus complicaciones, sin embargo esto no fue estudiado.

Del mismo modo, el resultado secundario carece de relevancia clínica (menor ganancia de peso, cercano a $1,5 \mathrm{~kg}$ ). Un resultado relevante hubiese sido reducción en la tasa de obesidad o sobrepeso, o reducción en la tasa de complicaciones asociadas al sobrepeso.

Un segundo aspecto crítico es la presencia de cointervenciones: la intervención estudiada es la dieta de bajo índice glicémico, sin embargo existen cointervenciones en el grupo de tratamiento, ya que además de la dieta reciben educación nutricional sobre alimentación saludable y refuerza el mantener la dieta durante todo el embarazo, lo que no se realiza en el grupo control.

El tercer aspecto deficiente es la falta de ciego: solo fueron ciegos los ecografistas, lo cual carece de relevancia ya que el resultado a medir es posnatal; hubiese sido mejor que los recolectores de datos, los que evalúan a los recién nacidos y los que analizan los datos fueran ciegos a la intervención.

Tabla I

RESULTADOS DEL ESTUDIO (1)

\begin{tabular}{|c|c|c|c|c|}
\hline Resultado & $\begin{array}{l}\text { Intervención } \\
\quad(n=372)\end{array}$ & $\begin{array}{l}\text { Control } \\
(n=387)\end{array}$ & $\begin{array}{l}\text { Diferencia promedio } \\
\text { (IC95\%) }\end{array}$ & Valor $\mathrm{p}$ \\
\hline \multicolumn{5}{|l|}{ Primario } \\
\hline Peso nacimiento & $4034(510)$ & $4006(497)$ & $\begin{array}{c}28,6 \\
(-45,6 \text { a } 102,8)\end{array}$ & 0,449 \\
\hline \multicolumn{5}{|l|}{ Secundarios } \\
\hline Ganancia de peso 24 sem (kg) & $5,3(2,7)$ & $5,(2,7)$ & $\begin{array}{c}-0,244 \\
(-0,786 \text { a } 0,299)\end{array}$ & 0,378 \\
\hline Ganancia de peso 28 sem (kg) & $7,1(2,8)$ & $7,7(3,0)$ & $\begin{array}{c}-0,593 \\
(-1,072 \text { a }-0,114)\end{array}$ & 0,015 \\
\hline Ganancia de peso 34 sem (kg) & $10,1(3,7)$ & $10,9(3,9)$ & $\begin{array}{c}-0,83 \\
(-1,48 a-0,182)\end{array}$ & 0,012 \\
\hline Ganancia de peso 40 sem (kg) & $12,2(4,4)$ & $13,7(4,9)$ & $\begin{array}{c}-1,346 \\
(-2,451 \text { a }-0,241)\end{array}$ & 0,017 \\
\hline
\end{tabular}

Valores son promedios (土DS). 
El éxito secundario reportado como la disminución de ganancia de peso durante el embarazo si fue significativo, pero al analizar más a fondo el dato se observa que esta diferencia es de $1,5 \mathrm{~kg}$, lo cual es poco relevante.

Validez externa: El estudio podría ser aplicable a nuestra población, ya que la prescripción de una dieta y la educación alimentaria son medidas de bajo costo. La exclusión de mujeres con antecedente de un recién nacido macrosómico, pero con diabetes gestacional, reduce la aplicabilidad externa de esta investigación. Las mujeres con diabetes pudieron ser incluidas y analizadas de modo separado.

\section{CONCLUSIONES}

Estudio de buena calidad (randomizado), sin embargo, con importantes fuentes potenciales de error: resultados primarios y secundarios clínicamente irrelevantes, presencia de cointervenciones y falta de ciego. Los resultados de esta investigación no resultan de utilidad para cambiar nuestra práctica clínica habitual y no deben ser considerados.

\section{REFERENCIAS}

1. Walsh JM, McGowan CA, Mahony R, Foley ME, McAuliffe FM. Low glycaemic index diet in pregnancy to prevent macrosomia (ROLO study): randomized control trial. BMJ 2012;345:e5605. doi: 10.1136/bmj. e5605. Hallado en: http://www.ncbi.nlm.nih.gov/pmc/ articles/PMC3431285/pdf/bmj.e5605.pdf

2. Chauhan SP, Grobman WA, Gherman RA, Chauhan VB, Chang G, Magann EF, Hendrix NW. Suspicion and treatment of the macrosomic fetus: a review. Am J Obstet Gynecol 2005;193:332-46.

3. Ju H, Chadha Y, Donovan T, O'Rourke P. Fetal macrosomia and pregnancy outcomes. Aust N Z J Obstet Gynaecol 2009;49:504-9.

4. Mahony R, Walsh C, Foley ME, Daly L, O'Herlihy C. Outcome of second delivery after prior macrosomic infant in women with normal glucose tolerance. Obstet Gynecol 2006;107:857-62.

5. Clapp JF 3rd. Maternal carbohydrate intake and pregnancy outcome. Proc Nutr Soc 2002;61:45-50.

6. Moses RG, Luebcke M, Davis WS, Coleman KJ, Tapsell LC, Petocz P, et al. Effect of a low-glycaemic-index diet during pregnancy on obstetric outcomes. Am J Clin Nutr 2006;84:807-12. 